\title{
Rational-Like Solutions of a Differential-Difference Equation Related to Ablowitz-Ladik Spectral Problem
}

\author{
Jiang-ping Zhang, ${ }^{1}$ Qi Li, ${ }^{1}$ and Shou-ting Chen ${ }^{2}$ \\ ${ }^{1}$ Department of Mathematics, East China Institute of Technology, Fuzhou 344000, China \\ ${ }^{2}$ Mathematics and Physical Sciences Technology, Xuzhou Institute of Technology, Xuzhou, Jiangsu 221008, China
}

Correspondence should be addressed to Qi Li; qli289@aliyun.com

Received 29 May 2015; Revised 11 August 2015; Accepted 12 August 2015

Academic Editor: Josè A. Tenereiro Machado

Copyright ( $\odot 215$ Jiang-ping Zhang et al. This is an open access article distributed under the Creative Commons Attribution License, which permits unrestricted use, distribution, and reproduction in any medium, provided the original work is properly cited.

By using the Casoratian technique, we construct the double Casoratian solutions whose entries satisfy matrix equation of a differential-difference equation related to the Ablowitz-Ladik spectral problem. Soliton solutions and rational-like solutions are obtained from taking special cases in general solutions.

\section{Introduction}

In recent decades, soliton equations have attracted much attention for both their physical and mathematical significance. For the soliton solutions of soliton equations, they scatter elastically with phase-shifts or amplitude changes in uniform or nonuniform media in dynamics. Many soliton equations such as $\mathrm{KdV}, \mathrm{mKdV}$, nonlinear Schrödinger equation, and sine-Gordon equation are viewed as the compatibility condition of the Ablowitz-Kaup-Newell-Segur (AKNS) spectral problem. Recently, the nonlinear Schrödinger equation attracts much attention in many fields such as oceanics, nonlinear optics, Bose-Einstein condensations [1], and atmospherics, for it interprets some freak wave (rogue wave) [2]. The propagation of the optical field complex envelope in a single-mode fiber, accounting for group velocity dispersion and Kerr nonlinearity, is governed by the nonlinear Schrödinger equation (see [3]). As the discrete version of the AKNS spectral problem, the Ablowitz-Ladik (AL) spectral problem has been studied for a few decades. The nonlinear self-dual network and Toda lattice equations are important equations related to the AL spectral problem. The Toda lattice is a system of unit masses, connected by nonlinear springs subject to an exponential restoring force [4]. The corresponding Ablowitz-Ladik hierarchy is researched for its symmetries [5] and its decompositions [6] in mathematical sense. It is interesting to study the differential-difference equation in the Ablowitz-Ladik hierarchy and find their soliton and rational solutions (see $[5,6]$ and references therein).

Many methods such as the inverse scattering transformation [7], Hirota method [8], Bäcklund transformation (e.g., [9]), dressing method (e.g., [10]), and Wronskian/Casoratian technique [11] can be used for finding their solutions of continuous [12] and discrete soliton equations [13] and soliton equations with self-consistent sources [14]. It is well known that the Wronskian/Casoratian technique has been used to construct various types of exact solutions of soliton equations, such as soliton solutions (e.g., [15]) and rational solutions [16]. N-soliton solution of the discrete-time relativistic Toda lattice equation is explicitly constructed in the form of the Casorati determinant [17]. The resulting solutions can be verified by direct substitution into the corresponding linear equation. By using the technique, the solitons and the rational solutions are introduced and constructed. In the paper, we would like to consider the differential-difference equation related to the Ablowitz-Ladik spectral problem [18]:

$$
\left(\begin{array}{c}
Q_{n} \\
R_{n}
\end{array}\right)_{t}=\left(\begin{array}{c}
Q_{n+1}\left(1-Q_{n} R_{n}\right) \\
-R_{n+1}\left(1-Q_{n} R_{n}\right)
\end{array}\right)
$$


which corresponding Lax pair is

$$
\begin{aligned}
\left(\begin{array}{l}
\phi_{1, n+1} \\
\phi_{2, n+1}
\end{array}\right) & =\left(\begin{array}{cc}
z & Q_{n} \\
R_{n} & \frac{1}{z}
\end{array}\right)\left(\begin{array}{c}
\phi_{1, n} \\
\phi_{2, n}
\end{array}\right), \\
\left(\begin{array}{l}
\phi_{1, n} \\
\phi_{2, n}
\end{array}\right)_{t} & =\left(\begin{array}{cc}
\frac{1}{2} z^{2}-Q_{n} R_{n-1} & z Q_{n} \\
z R_{n-1} & -\frac{1}{2} z^{2}
\end{array}\right)\left(\begin{array}{l}
\phi_{1, n} \\
\phi_{1, n}
\end{array}\right),
\end{aligned}
$$

where $Q_{n}=Q(t, n), R_{n}=R(t, n), \phi_{1, n}=\phi_{1}(t, n)$, and $\phi_{2, n}=\phi_{2}(t, n)$ are functions dependent on variable $t, n(n \in \mathbb{Z})$ and decrease rapidly when $|n|$ tend to $\infty$ and $z$ is a spectral parameter. In general, the distinct discrete eigenvalues $\left\{z_{k}\right\}$ are included in the scattering data of direct scattering problem in the frame of the inverse scattering transform. $\left\{z_{k}\right\}$ usually influence the shape and propagation of the multisolitons.

The paper is organized as follows. Section 2 gives the double Casoratian solution of (1). In Section 3 soliton solutions and "rational-like" solutions are derived from general solutions directly.

\section{The Double Casoratian Solution}

By the dependent variable transformation

$$
\begin{aligned}
& Q_{n}=\frac{g_{n}}{f_{n}}, \\
& R_{n}=\frac{h_{n}}{f_{n}},
\end{aligned}
$$

(1) can be transformed to bilinear form

$$
\begin{aligned}
\left(e^{D_{n}}-1\right) f_{n} \cdot f_{n} & =-g_{n} h_{n}, \\
D_{t} g_{n} \cdot f_{n} & =g_{n+1} f_{n-1}, \\
D_{t} h_{n} \cdot f_{n} & =-h_{n-1} f_{n+1},
\end{aligned}
$$

where the operators $D_{t}$ and $e^{D_{n}}$ are defined by

$$
\begin{aligned}
D_{t} f(t, n) \cdot g(t, n) & =\left.\left(\partial_{t}-\partial_{t^{\prime}}\right) f(t, n) g(t, n)\right|_{t=t^{\prime}}, \\
\partial_{t}=\frac{\partial}{\partial t}, & \\
e^{D_{n}} f(t, n) \cdot g(t, n) & =f(t, n+1) g(t, n-1) .
\end{aligned}
$$

To use the Casoratian technique, we give the compact notation by Freeman and Nimmo [11]:

$$
\begin{aligned}
\operatorname{Cas}^{N, M}\left(\phi_{n}, \psi_{n}\right) \\
=\left|\phi_{n}, E \phi_{n}, \ldots, E^{N-1} \phi_{n} ; \psi_{n}, E \psi_{n}, \ldots, E^{M-1} \psi_{n}\right| \\
=|\widehat{N-1} ; \widehat{M-1}|,
\end{aligned}
$$

where

$$
\begin{aligned}
& \phi_{n}=\left(\phi_{1, n}, \phi_{2, n}, \ldots, \phi_{N+M, n}\right)^{T}, \\
& \psi_{n}=\left(\psi_{1, n}, \psi_{2, n}, \ldots, \psi_{N+M, n}\right)^{T} .
\end{aligned}
$$

$E$ is shift operator defined by $E \phi_{n}=\phi_{n+1}$. We also denote that

$$
\begin{aligned}
& |-1, \widehat{N-1} ;-1, \widehat{M-1}|=\mid E^{-1} \phi_{n}, \phi_{n}, E \phi_{n}, \ldots, \\
& \quad E^{N-1} \phi_{n} ; E^{-1} \psi_{n}, \psi_{n}, E \psi_{n}, \ldots, E^{M-1} \psi_{n} \mid, \\
& |\widehat{2 N-2} ; \widehat{2 M-2}|=\mid \phi_{n}, E^{2} \phi_{n}, \ldots, E^{2 N-2} \phi_{n} ; \psi_{n}, E^{2} \psi_{n}, \\
& \quad \ldots, E^{2 M-2} \psi_{n} \mid, \\
& |\widehat{2 N-1} ; \widehat{2 M-1}|=\mid E \phi_{n}, E^{3} \phi_{n}, \ldots, E^{2 N-1} \phi_{n} ; E \psi_{n}, \\
& \quad E^{3} \psi_{n}, \ldots, E^{2 M-1} \psi_{n} \mid,
\end{aligned}
$$

where $E^{-1} \phi_{n}=\phi_{n-1}$. It is easy to get the following lemma.

Lemma 1. Consider the following:

$$
\begin{aligned}
& |Q, a, b||Q, c, d|-|Q, a, c||Q, b, d|+|Q, a, d||Q, b, c| \\
& \quad=0
\end{aligned}
$$

where $Q$ is an $N \times(N-2)$ matrix and $a, b, c$, and d represent $N$-dimensional column vectors.

Theorem 2. Equation (1) has the double Casoratian solution,

$$
\begin{aligned}
& f_{n}=|\widehat{2 N-2} ; \widehat{2 M-2}|, \\
& g_{n}=|-1, \widehat{2 N-1} ; \widehat{2 M-3}|, \\
& h_{n}=-|\widehat{2 N-3} ;-1, \widehat{2 M-1}|,
\end{aligned}
$$

for the condition equations,

$$
\begin{aligned}
\phi_{j, n+1} & =e^{k_{j}} \phi_{j, n}, \\
\psi_{j, n-1} & =e^{k_{j}} \psi_{j, n}, \\
\phi_{j, n, t} & =\frac{1}{2} \phi_{j, n+2}, \\
\psi_{j, n, t} & =-\frac{1}{2} \psi_{j, n-2}
\end{aligned}
$$

$$
(j=1,2, \ldots, N+M)
$$

and in general

$$
\begin{aligned}
\phi_{n+1} & =A \phi_{n}, \\
\psi_{n-1} & =A \psi_{n}, \\
\phi_{n, t} & =\frac{1}{2} \phi_{n+2}, \\
\psi_{n, t} & =-\frac{1}{2} \psi_{n-2},
\end{aligned}
$$


where $A=\left(a_{i j}\right)_{(N+M) \times(N+M)}$ is an arbitrary matrix independent of $t$ and $n$. Thus the corresponding solution of (1) can be expressed as

$$
\begin{aligned}
& Q_{n}=\frac{|-1, \widehat{2 N-1} ; \widehat{2 M-3}|}{|\widehat{2 N-2} ; \widehat{2 M-2}|}, \\
& R_{n}=-\frac{|\widehat{2 N-3} ;-1, \widehat{2 M-1}|}{|\widehat{2 N-2} ; \widehat{2 M-2}|} .
\end{aligned}
$$

Proof. Here we give two notations. $(\widetilde{2 N-2} ; \widetilde{2 M-2})$ denotes the columns $\left(E^{2} \phi_{n}, E^{4} \phi_{n}, \ldots, E^{2 N-2} \phi_{n} ; E^{2} \psi_{n}, E^{4} \psi_{n}, \ldots\right.$, $\left.E^{2 M-2} \psi_{n}\right)$, which is related to the even-order derivatives. $(\widetilde{2 N-1} ; 2 \overparen{2 M-1})$ denotes the columns $\left(E^{3} \phi_{n}, E^{5} \phi_{n}, \ldots\right.$, $\left.E^{2 N-1} \phi_{n} ; E^{3} \psi_{n}, E^{5} \psi_{n}, \ldots, E^{2 M-1} \psi_{n}\right)$, which is related to the odd-order derivatives. First, by use of the Casoratian technique, it is easy to get

$$
\begin{aligned}
f_{n+1} & =|\widehat{2 N-1} ; \widehat{2 M-1}|, \\
f_{n-1} & =|-1, \widehat{2 N-3} ;-1, \widehat{2 M-3}|, \\
g_{n+1} & =|\widehat{2 N} ; \widehat{2 M-2}|, \\
h_{n-1} & =-|\widehat{2 N-4} ;-2, \widehat{2 M-2}|, \\
f_{n, t} & =\frac{1}{2}(|\widehat{2 N-4}, 2 N ; \widehat{2 M-2}| \\
& -|\widehat{2 N-2} ;-2, \widehat{2 M-2}|), \\
g_{n, t} & =\frac{1}{2}(|-1, \widehat{2 N-3}, 2 N+1 ; \widehat{2 M-3}| \\
& -|-1, \widehat{2 N-1} ;-1, \widehat{2 M-3}|), \\
h_{n, t} & =\frac{1}{2}(|\widehat{2 N-3} ;-3, \widehat{2 M-1}| \\
& -\mid \widehat{2 N-5,2 N-1 ;-1, \widehat{2 M-1} \mid) .}
\end{aligned}
$$

For (4a), note that

$$
\begin{aligned}
& |\widehat{2 N-2} ; \widehat{2 M-2}|^{2}=\prod_{j=1}^{N+M} e^{k_{j}}|\widehat{2 N-2} ; \widehat{2 M-2}| \\
& \quad \cdot \prod_{j=1}^{N+M} e^{-k_{j}}|\widehat{2 N-2} ; \widehat{2 M-2}| \\
& =|\widehat{2 N-1} ;-1, \widehat{2 M-3}||-1, \widehat{2 N-3} ; \widehat{2 M-1}|
\end{aligned}
$$

and taking $Q=(\widehat{2 N-3}, \widehat{2 M-3})$ in (9) yields

$$
\begin{aligned}
& |\widehat{2 N-3}, 2 N-1 ; \widehat{2 M-3}, 2 M-1| \\
& \cdot|-1, \widehat{2 N-3} ;-1, \widehat{2 M-3}| \\
& -|\widehat{2 N-3}, 2 N-1 ;-1, \widehat{2 M-3}| \\
& \cdot|-1, \widehat{2 N-3} ; \widehat{2 M-3}, 2 M-1| \\
& -|-1, \widehat{2 N-3}, 2 N-1 ; \widehat{2 M-3}| \\
& \cdot|\widehat{2 N-3} ;-1, \widehat{2 M-3}, 2 M-1|=0 .
\end{aligned}
$$

It follows that

$$
\begin{aligned}
f_{n}^{2}- & g_{n} h_{n}-f_{n+1} f_{n-1} \\
= & |\widehat{2 N-1} ;-1, \widehat{2 N-3}||-1, \widehat{2 N-3} ; \widehat{2 M-1}| \\
& \quad+|-1, \widehat{2 N-1} ; \widehat{2 M-3}||-1, \widehat{2 N-3} ; \widehat{2 M-1}| \\
& \quad-|\widehat{2 N-1} ; \widehat{2 M-1}||-1, \widehat{2 N-3} ;-1, \widehat{2 M-3}| \\
= & 0 .
\end{aligned}
$$

Thus, ((10a), (10b), (10c)) solves (4a).

For (4b), from (9) and noting the following identities,

$$
\prod_{j=1}^{N+M} e^{k_{j}}|\widehat{2 N-2} ; \widehat{2 M-2}|=|\widehat{2 N-1} ;-1, \widehat{2 M-3}|,
$$

$$
\begin{aligned}
& \prod_{j=1}^{N+M} e^{k_{j}}|\widehat{2 N-4}, 2 N ; \widehat{2 M-2}| \\
& \quad=|\widehat{2 N-3}, 2 N+1 ;-1, \widehat{2 M-3}|, \\
& \prod_{j=1}^{N+M} e^{k_{j}}|\widehat{2 N} ; \widehat{2 M-2}|=|\widehat{2 N+1} ; \widehat{2 M-3}|,
\end{aligned}
$$

we have

$$
\begin{aligned}
|-1, \widehat{2 N-3}, 2 N+1 ; \widehat{2 M-3}||\widehat{2 N-2} ; \widehat{2 M-2}| \\
-|-1, \widehat{2 N-1} ; \widehat{2 M-3}||\widehat{2 N-4}, 2 N ; \widehat{2 M-2}| \\
-|\widehat{2 N} ; \widehat{2 M-2}||-1, \widehat{2 N-3} ;-1, \widehat{2 M-3}|=0 .
\end{aligned}
$$

Similarly, we can get

$$
\begin{aligned}
-|-1, \widehat{2 N-1} ;-1, \widehat{2 M-3}||| \widehat{2 N-2} ; \widehat{2 M-2} \mid \\
\quad+|-1, \widehat{2 N-1} ; \widehat{2 M-3}||| \widehat{2 N-2} ;-2, \widehat{2 M-2} \mid \\
-|\widehat{2 N} ; \widehat{2 M-2}||-1, \widehat{2 N-3} ;-1, \widehat{2 M-3}|=0 .
\end{aligned}
$$


By combining (19) with (20) and from ((14a), (14b), (14c), (14d), (14e)), one can have that ((10a), (10b), (10c)) solves (4b). In a similar way, we can also verify that ((10a), (10b), (10c)) solves $(4 \mathrm{c})$.

Now from ((10a), (10b), (10c)) and using the Casoratian technique, we show that ((10a), (10b), (10c)) solves ((4a), (4b), (4c)) for the condition equations ((12a), (12b)). In fact, we only need to verify (15), which can be obtained by the following identities:

$$
\begin{aligned}
& |\widehat{2 N-1} ;-1, \widehat{2 M-3}|=|A||\widehat{2 N-2} ; \widehat{2 M-2}|, \\
& |-1, \widehat{2 N-3} ; \widehat{2 M-1}|=\left|A^{-1}\right||\widehat{2 N-2} ; \widehat{2 M-2}|,
\end{aligned}
$$

where $A^{-1}$ is the inverse matrix of $A$.

\section{Soliton Solutions and Rational-Like Solutions}

From ((12a), (12b)), we can get the general solution

$$
\begin{aligned}
& \phi_{n}=C e^{(1 / 2) e^{2 B} t+n B}, \\
& \psi_{n}=D e^{-(1 / 2) e^{2 B} t-n B},
\end{aligned}
$$

where $A=e^{B}, C=\left(c_{1}, c_{2}, \ldots, c_{N+M}\right)^{T}$, and $D=\left(d_{1}, d_{2}, \ldots\right.$, $\left.d_{N+M}\right)^{T}$ are real constant vectors. In order to get the soliton solutions and rational-like solutions, we take matrix $B$ in canonical form $\Gamma$ in (22) and expand the functions $\phi_{n}$ and $\psi_{n}$ :

$$
\begin{aligned}
& \phi_{n}=C e^{(1 / 2) e^{2 \Gamma} t+n \Gamma}=\sum_{s=0}^{\infty} P_{s}(n, t) e^{(1 / 2) t I} \Gamma^{s} C, \\
& \psi_{n}=D e^{-(1 / 2) e^{2 \Gamma} t-n \Gamma}=\sum_{s=0}^{\infty} P_{s}(-n,-t) e^{-(1 / 2) t I} \Gamma^{\mathcal{S}} D,
\end{aligned}
$$

where

$$
\begin{aligned}
P_{s}(n, t) & =\sum_{j=0}^{s} \frac{n^{j}}{j !} p_{s-j}(\widetilde{t}), \\
p_{s}(\widetilde{t}) & =\sum_{\|\gamma\|=s} \frac{2^{\|\gamma\|}}{\gamma ! \widetilde{t}^{\gamma}}, \\
\gamma & =\left(\gamma_{1}, \gamma_{2}, \gamma_{3}, \ldots\right), \quad \gamma_{j} \geq 0,(j=1,2,3, \ldots), \\
\|\gamma\| & =\gamma_{1}+2 \gamma_{2}+3 \gamma_{3}+\cdots, \\
\gamma ! & =\gamma_{1} ! \gamma_{2} ! \gamma_{3} ! \ldots, \\
\tilde{t} & =\left(\frac{t_{1}}{2}, \frac{t_{2}}{2}, \frac{t_{3}}{2}, \ldots\right), \\
t_{j} & =\frac{t}{j !}, \\
\tilde{t}^{\gamma} & =\left(\frac{t_{1}}{2}\right)^{\gamma_{1}}\left(\frac{t_{2}}{2}\right)^{\gamma_{2}}\left(\frac{t_{3}}{2}\right)^{\gamma_{3}} \ldots
\end{aligned}
$$

and $I$ is the unit matrix. If

$$
\Gamma=\left(\begin{array}{cccc}
k_{1} & & & 0 \\
& k_{2} & & \\
& & \ddots & \\
0 & & & k_{N+M}
\end{array}\right), \quad k_{i} \neq k_{j}(i \neq j)
$$

then we can obtain the soliton solutions (13) from (22) (substituting $B$ for $\Gamma$ ), where

$$
\begin{aligned}
& \phi_{j, n}=c_{j} e^{(1 / 2) e^{2 k_{j}} t+n k_{j}} \\
& \psi_{j, n}=d_{j} e^{-(1 / 2) e^{2 k_{j}} t-n k_{j}}
\end{aligned}
$$

$$
(j=1,2, \ldots, N+M)
$$

If

$$
\Gamma=\left(\begin{array}{cccc}
0 & & & 0 \\
1 & 0 & & \\
& \ddots & \ddots & \\
0 & & 1 & 0
\end{array}\right)_{N+M}
$$

then it follows from ((23a), (23b)) and $\Gamma^{N+M}=0$ that

$$
\begin{aligned}
& \phi_{n}=\sum_{s=0}^{N+M-1} P_{s}(n, t) e^{(1 / 2) t I} \Gamma^{s} C, \\
& \psi_{n}=\sum_{s=0}^{N+M-1} P_{s}(-n,-t) e^{-(1 / 2) t I} \Gamma^{s} D .
\end{aligned}
$$

We specially give some rational-like solutions in the following. Taking $(N, M)=(1,1), \Gamma=\left(\begin{array}{ll}0 & 0 \\ 1 & 0\end{array}\right)$, and $C=D=(1,0)^{T}$ in $((28 \mathrm{a}),(28 \mathrm{~b}))$ leads to

$$
\begin{aligned}
& \phi_{n}=e^{(1 / 2) t}\left(\begin{array}{c}
1 \\
n+t
\end{array}\right), \\
& \psi_{n}=e^{-(1 / 2) t}\left(\begin{array}{c}
1 \\
-n-t
\end{array}\right) .
\end{aligned}
$$

Hence, the solution ((10a), (10b), (10c)) reads

$$
\begin{aligned}
& Q_{n}=-\frac{e^{t}}{n+t}, \\
& R_{n}=-\frac{e^{-t}}{n+t} .
\end{aligned}
$$


Similarly, we can calculate the following rational-like solutions with respect to $n$ from ((28a), (28b)),

$$
\begin{aligned}
& Q_{n}=\frac{2 e^{t}}{n+n^{2}+2 n t+t^{2}}, \\
& R_{n}=-\frac{e^{-t}\left(n+n^{2}+2 n t+2 t+t^{2}\right)}{n+n^{2}+2 n t+t^{2}} \\
& Q_{n}=-\frac{e^{t}\left(n+n^{2}+2 n t+t^{2}\right)}{n+n^{2}+2 n t+2 t+t^{2}} \\
& R_{n}=-\frac{2 e^{-t}}{n+n^{2}+2 n t+2 t+t^{2}} \\
& Q_{n}=-\frac{2 e^{t}\left[n^{3}+3 n^{2}+3 n^{2} t+t^{3}+n\left(2+3 t+3 t^{2}\right)\right]}{n^{4}+4 n^{3}(t+1)+t^{2}\left(6+4 t+t^{2}\right)+n^{2}\left(5+12 t+6 t^{2}\right)+2 n\left(1+4 t+6 t^{2}+2 t^{3}\right)}, \\
& R_{n}=\frac{2 e^{-t}\left[n^{3}+3 n^{2}(t+1)+t\left(6+6 t+t^{2}\right)+n\left(2+9 t+3 t^{2}\right)\right]}{n^{4}+4 n^{3}(t+1)+t^{2}\left(6+4 t+t^{2}\right)+n^{2}\left(5+12 t+6 t^{2}\right)+2 n\left(1+4 t+6 t^{2}+2 t^{3}\right)} \\
& Q_{n}=-\frac{6 e^{t}}{n^{3}+t^{3}+3 n^{2}(t+1)+n\left(2+3 t+3 t^{2}\right)}, \\
& R_{n}=-\frac{e^{-t}\left[n^{4}+n^{3}(t+1)+n^{2}\left(5+12 t+6 t^{2}\right)+t^{2}\left(6+4 t+t^{2}\right)+2 n\left(1+4 t+6 t^{2}+2 t^{3}\right)\right]}{2\left[n^{3}+t^{3}+3 n^{2}(t+1)+n\left(2+3 t+3 t^{2}\right)\right]} ; \\
& Q_{n}=-\frac{e^{t}\left[n^{4}+n^{3}(t+1)+n^{2}\left(5+12 t+6 t^{2}\right)+t^{2}\left(6+4 t+t^{2}\right)+2 n\left(1+4 t+6 t^{2}+2 t^{3}\right)\right]}{n^{3}+t\left(6+6 t+t^{2}\right)+3 n^{2}(t+1)+n\left(2+9 t+3 t^{2}\right)}, \\
& R_{n}=-\frac{6 e^{-t}}{n^{3}+t\left(6+6 t+t^{2}\right)+3 n^{2}(t+1)+n\left(2+9 t+3 t^{2}\right)}
\end{aligned}
$$

for $(N, M)=(2,1),(N, M)=(1,2),(N, M)=(2,2)$, $(N, M)=(3,1)$, and $(N, M)=(1,3)$, respectively.

\section{Conclusion}

The differential-difference equation related to the AblowitzLadik spectral problem, which has mathematical and physical significance, is solved by the Casoratian technique. The soliton and rational-like solutions are obtained through some condition equations in general case, which can be examined by substituting the solutions into the Casoratian solutions directly.

\section{Conflict of Interests}

The authors declare that there is no conflict of interests regarding the publication of this paper.

\section{Acknowledgments}

This project is supported by the National Natural Science Foundation of China (nos. 11301454 and 11271168), the
Natural Science Foundation of Jiangxi Province of China (no. 20142BAB201006), the Research Foundation of Education Bureau of Jiangxi Province of China (no. GJJ13459), the Natural Science Foundation of the Colleges and Universities of Jiangsu Province (no. 13KJD110009), and the Graduate Innovation Foundation of East China Institute of Technology.

\section{References}

[1] B. L. Guo, "Nonlinear Schrödinger equation (I): Bose-Einstein condensation and rogue waves," Advances in Mathematics, vol. 40, no. 4, pp. 393-399, 2011 (Chinese).

[2] N. Akhmediev and A. Ankiewicz, "Modulation instability, Fermi-Pasta-Ulam recurrence, rogue waves, nonlinear phase shift, and exact solutions of the Ablowitz-Ladik equation," Physical Review E, vol. 83, Article ID 046603, 2011.

[3] G. P. Agrawal, Nonlinear Fiber Optics, Academic Press, 2007.

[4] M. Toda, "Waves in nonlinear lattice," Progress of Theoretical Physics Supplement, vol. 45, pp. 174-200, 1970.

[5] D.-J. Zhang and S.-T. Chen, "Symmetries for the AblowitzLadik hierarchy: part I. Four-potential case," Studies in Applied Mathematics, vol. 125, no. 4, pp. 393-418, 2010. 
[6] X. Geng, H. H. Dai, and J. Zhu, "Decomposition of the discrete Ablowitz-Ladik hierarchy," Studies in Applied Mathematics, vol. 118, no. 3, pp. 281-312, 2007.

[7] M. J. Ablowitz and P. A. Clarkson, Solitons, Nonlinear Evolution Equations and Inverse Scattering, Cambridge University Press, 1991.

[8] R. Hirota, "Exact solution of the korteweg-de vries equation for multiple Collisions of solitons," Physical Review Letters, vol. 27, article 1192, 1971.

[9] Y.-P. Sun and H.-W. Tam, "New type of nonisospectral KP equation with self-consistent sources and its bilinear Bäcklund transformation," Journal of Nonlinear Mathematical Physics, vol. 18, no. 2, pp. 323-336, 2011.

[10] Y. Q. Yao, X. J. Liu, and Y. B. Zeng, "A new extended discrete KP hierarchy and a generalized dressing method," Journal of Physics A: Mathematical and Theoretical, vol. 42, no. 45, Article ID 454026, 2009.

[11] N. C. Freeman and J. J. C. Nimmo, "Soliton solutions of the Korteweg de Vries and the Kadomtsev-Petviashvili equations: the Wronskian technique," Proceedings of the Royal Society of London A: Mathematical, Physical and Engineering Sciences, vol. 389, no. 1797, pp. 319-329, 1983.

[12] S.-F. Deng, D.-Y. Chen, and D.-J. Zhang, "The multisoliton solutions of the KP equation with self-consistent sources," Journal of the Physical Society of Japan, vol. 72, no. 9, pp. 21842192, 2003.

[13] J. J. Nimmo, "Soliton solution of three differential-difference equations in Wronskian form," Physics Letters A, vol. 99, no. 6-7, pp. 281-286, 1983.

[14] Q. Li, D. J. Zhang, and D. Y. Chen, "Solving the hierarchy of the nonisospectral $\mathrm{KdV}$ equation with self-consistent sources via the inverse scattering transform," Journal of Physics A: Mathematical and Theoretical, vol. 41, Article ID 355209, 2008.

[15] Q. Li, Q. Duan, and J. Zhang, "Exact multisoliton solutions of general nonlinear Schrödinger equation with derivative," The Scientific World Journal, vol. 2014, Article ID 593983, 7 pages, 2014.

[16] W. X. Ma and Y. C. You, "Rational solutions of the Toda lattice equation in Casoratian form," Chaos, Solitons \& Fractals, vol. 22, no. 2, pp. 395-406, 2004.

[17] K.-I. Maruno, M. Oikawa, and K. Kajiwara, "Casorati determinant solution for the discrete-time relativistic Toda lattice equation," Physics Letters A, vol. 241, no. 6, pp. 335-343, 1998.

[18] M. J. Ablowitz and J. F. Ladik, "Nonlinear differential-difference equations," Journal of Mathematical Physics, vol. 16, pp. 598-603, 1975. 


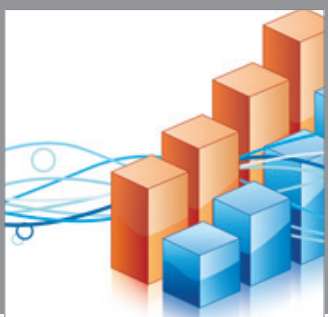

Advances in

Operations Research

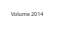

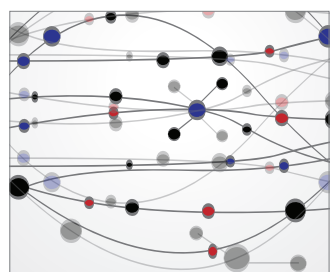

\section{The Scientific} World Journal
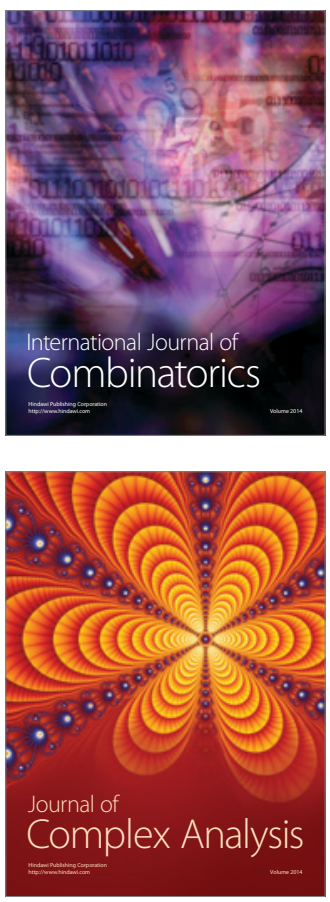

International Journal of

Mathematics and

Mathematical

Sciences
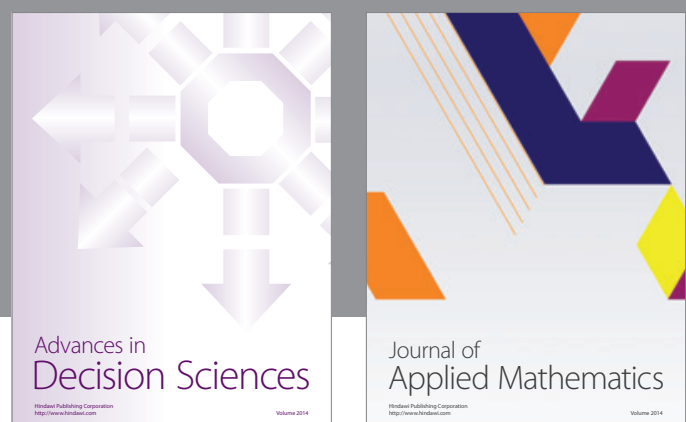

Journal of

Applied Mathematics
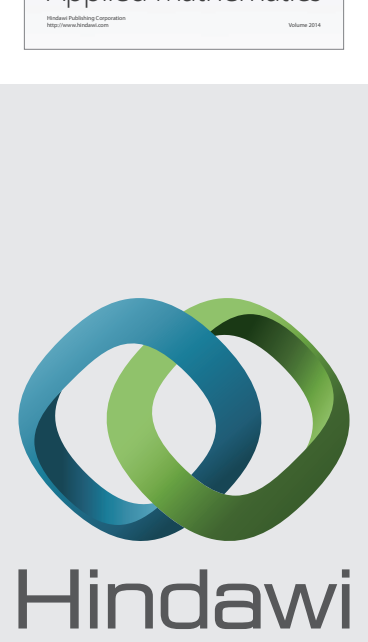

Submit your manuscripts at http://www.hindawi.com
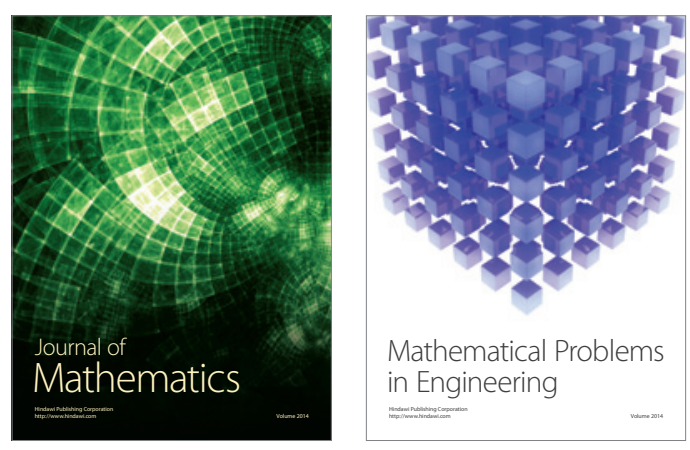

Mathematical Problems in Engineering
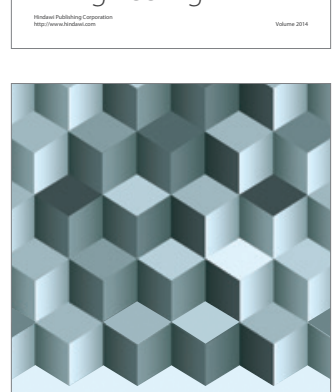

Journal of

Function Spaces
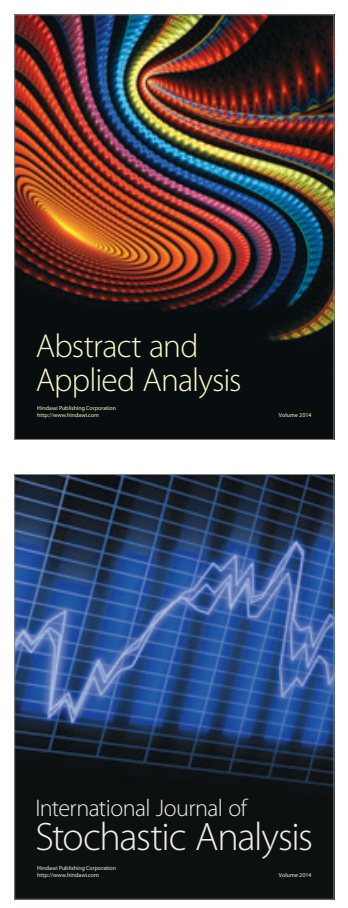

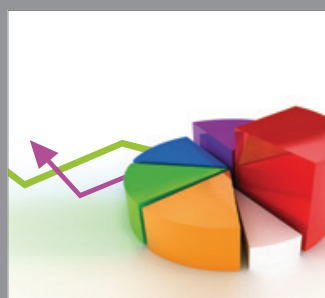

ournal of

Probability and Statistics

Promensencen
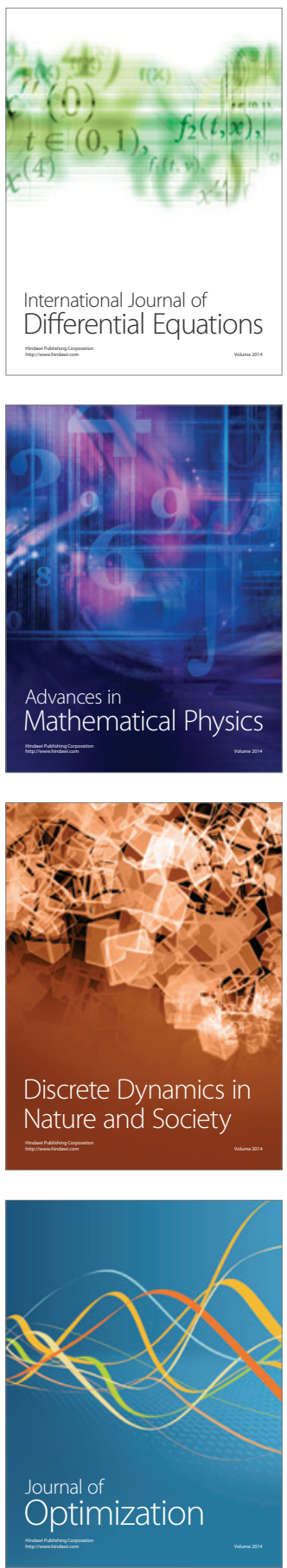